\title{
Masers in AGN environments
}

\author{
L. J. Greenhill \\ Harvard-Smithsonian Center for Astrophysics, 60 Garden St, Cambridge, MA 02138 USA \\ email: greenhill@cfa.harvard.edu
}

\begin{abstract}
Galactic nuclei are well known sources of $\mathrm{OH}$ and $\mathrm{H}_{2} \mathrm{O}$ maser emission. It appears that intense star formation in ultra-luminous infrared galaxies drives most $\mathrm{OH}$ sources. In contrast, nuclear activity appears to drive most $\mathrm{H}_{2} \mathrm{O}$ sources. When $\mathrm{H}_{2} \mathrm{O}$ emission originates in accretion disk structures, constrained geometry and dynamics enable robust interpretation of spectroscopic and imaging data. The principal science includes study of AGN geometry at parsec and sub-parsec radii and measurement of geometric distances in the Hubble Flow. New high accuracy estimates of the "Hubble constant, $\mathrm{H}_{\circ}$ " obtained from maser distances may enable new substantively improved constraints on fundamental cosmological parameters (e.g., dark energy).
\end{abstract}

Keywords. masers, accretion, galaxies: active, galaxies: Seyfert, cosmological parameters, distance scale, radio lines: galaxies

\section{1. $\mathrm{OH}$ and $\mathrm{H}_{2} \mathrm{O}$ masers in AGN}

Molecular emission is widespread among active galactic nuclei (e.g., Henkel et al. 1990, García-Burillo et al. 2007, and references therein). However, maser action is comparatively rare, having been detected only for $\mathrm{OH}$ and $\mathrm{H}_{2} \mathrm{O}$.

In general, $\mathrm{OH}$ maser sources lie in luminous infrared galaxies. Seven have been resolved on scales $\lesssim 100 \mathrm{pc}$. Most are associated at least in part with inclined circumnuclear disks, inferred from position-velocity gradients or limb brightened structures (see review by Pihlström 2005b and case studies by Momjian et al. 2006; Pihlström et al. 2001, 2005a; Richards et al. 2005; Rovilos et al. 2003; Trotter et al. 1997; Yates et al. 2000). Estimated radii are $10^{1}$ to $10^{2} \mathrm{pc}$ and dynamical masses are $10^{6}$ to $10^{8} \mathrm{M}_{\odot}$. However, connections between these structures and nuclear activity have yet to be demonstrated. Observational hurdles include separating star forming and AGN processes, and identifying direct links between the $\mathrm{OH}$ structures and accretion or outflow (e.g., Vignali et al. 2005; Klöckner \& Baan 2005; Baan \& Klöckner 2006).

In contrast, connections between structures traced by $\mathrm{H}_{2} \mathrm{O}$ masers and nuclear activity are reasonably well established. Water maser host galaxies are in nearly every case readily identified as AGN; luminosity from star formation is secondary. ( $\mathrm{A}$ few $\mathrm{H}_{2} \mathrm{O}$ masers are seen in star forming galaxies - Henkel et al. 2005; Greenhill 2002a, and references therein - and five $\mathrm{H}_{2} \mathrm{O}$ hosts exhibit $\mathrm{OH}$ emission as well (Gallimore et al. 1996; Tarchi et al. 2007) - but these constitute a small minority.) Where $\mathrm{H}_{2} \mathrm{O}$ emission has been mapped, and the underlying structures have been resolved, they are typically found to lie a few tenths to a few parsecs from the central engines, and to exhibit dynamical signatures characteristic of accretion and outflow.

In light of greater understanding of the association between $\mathrm{H}_{2} \mathrm{O}$ masers and AGN processes, the remainder of this review will focus on $\mathrm{H}_{2} \mathrm{O}$, leaving the complex and rapidly evolving story of $\mathrm{OH}$ to treatment elsewhere in this volume. 


\section{History}

Early interpretations of extragalactic $\mathrm{H}_{2} \mathrm{O}$ maser emission in nuclei (mistakenly) focused on star formation as driver. It was a familiar context. The NGC $4945 \mathrm{H}_{2} \mathrm{O}$ maser was the first discovered in a nucleus (Table 1), prior to identification of an AGN therein (Dos Santos \& Lépine 1979). The second nuclear $\mathrm{H}_{2} \mathrm{O}$ source was in a well-known Seyfert galaxy, Circinus, but this too was attributed to star formation (Gardner \& Whiteoak 1982). Claussen \& Lo (1986) delivered the first strong challenge, with evidence that nuclear $\mathrm{H}_{2} \mathrm{O}$ maser emission could be compact on scales of 3-10 pc and in close proximity to central engines. In light of the proposed unification paradigm for AGN, Claussen \& Lo conjectured that the emission arose from warm gas in the now canonical torus. Nearly a decade later, studies of NGC 4258 were the first to demonstrate that the emission in fact originates from annuli in the still more compact, relatively thin disks at radii on the order of 0.1 to $1 \mathrm{pc}$ (Miyoshi et al. 1995; Greenhill et al. 1995). Two additional contexts for maser emission were identified later: (1) downstream from central engines in association with radio jets (Gallimore et al. 1996; Claussen et al. 1998; Sawada-Satoh, this meeting) and (2) wide-angle outflows of AGN narrow-line regions (Greenhill et al. 2003a).

Table 1. Historical Perspective

\begin{tabular}{|c|c|c|c|}
\hline Date $^{(a)}$ & Event & Target & Reference \\
\hline $11 / 76$ & First extragalactic $\lambda 1 \mathrm{~cm} \mathrm{H}_{2} \mathrm{O}$ maser & M 33 & 1 \\
\hline $09 / 77$ & First $\mathrm{H}_{2} \mathrm{O}$ maser in a galactic nucleus & NGC $4945^{(b)}$ & 2 \\
\hline $04 / 82$ & First $\mathrm{H}_{2} \mathrm{O}$ maser in a known AGN & Circinus & 3 \\
\hline $09 / 83$ & Detection of compact structure with VLA \& VLBI & JGC1068/4258 & 4 \\
\hline $10 / 84$ & & NGC 4258 & 5 \\
\hline $01 / 92$ & $\begin{array}{l}-- \text { First Lull in Detection of New Masers - - } \\
\text { High-velocity emission discovered }\end{array}$ & NGC 4258 & 6 \\
\hline $03 / 93$ & Narrow-band surveys target optical AGN & & 7,8 \\
\hline $10 / 94$ & Demonstration that masers can trace accretion disks & NGC 4258 & 9 \\
\hline $04 / 94$ & & & 10 \\
\hline $08 / 95$ & Demonstration that masers can trace radio jets & NGC 1068 & 11 \\
\hline $11 / 95$ & & NGC 1052 & 12 \\
\hline $06 / 97$ & $\begin{array}{l}\text { Demonstration that masers can trace AGN winds } \\
\end{array}$ & & 13 \\
\hline $12 / 01$ & Wide-band surveys begin at DSN $70 \mathrm{~m}$ antennas & & 14,15 \\
\hline $04 / 02$ & Wide-band surveys begin at GBT 100m antenna & & 16 \\
\hline $12 / 03$ & HST program begins to X-calibrate Cepheid/maser distances & & 17 \\
\hline $01 / 05$ & First maser discovered in a quasar $(z \sim 0.7)$ & $\mathrm{J} 0804+3607$ & 18 \\
\hline $03 / 05$ & First submm $\mathrm{H}_{2} \mathrm{O}$ maser discovered in an AGN & NGC 3079 & 19 \\
\hline $11 / 06$ & Water Maser Cosmology Project begins (NRAO/CfA/MPI) & & 20 \\
\hline
\end{tabular}

(a) Observing dates associated with events in column 2 .

(b) Lépine \& Dos Santos (1977) reported an earlier detection possibly toward the nucleus of NGC 253, but independent studies failed to confirm it. See review by Greenhill (2002a).

References: (1) Churchwell et al. (1977), (2) Dos Santos \& Lépine (1979), (3) Gardner \& Whiteoak (1982), (4) Claussen \& Lo (1986), (5) Claussen et al. (1988), (6) Nakai, Inoue, \& Miyoshi (1993), (7) Braatz et al. (1996), (8) Greenhill et al. (2002b), (9) Greenhill et al. (1995), (10) Miyoshi et al (1995), (11) Gallimore et al. (1996), (12) Claussen et al. (1998), (13) Greenhill et al. (2003a), (14) Greenhill et al. (2003b), (15) Kondratko et al. (2006a), (16) Braatz et al. (2004), (17) Macri et al. (2006), (18) Barvainis \& Antonucci (2005), (19) Humphreys et al. (2005), and (20) Braatz et al. (this volume). 


\section{Surveys}

Water maser sources in nuclei are rare. The historical record of search programs is full of hypothesized correlations and physical arguments intended to identify selection criteria that would enable detection rates $\gg$ a few percent. Though small samples have achieved detection rates as large as $\sim 50 \%$ (Henkel et al. 2005), no large survey $\left(\mathrm{N}>10^{2}\right.$ ) has exceeded 1-10\%. The most consistently successful sample is optically identified AGN, as first pursued in the mid-1990s (e.g., Braatz et al. 1996). Nearly all masers lie in Seyfert 2 objects and Low Ionization Nuclear Emission Regions (LINERs), though some are known to lie in ostensibly inactive galaxies and transition objects between Seyfert 1 and 2. None are known to lie in "pole-on" active systems. The incidence rate of maser emission among known Seyfert-2 objects and LINERs is on the order of $20 \%$ for $c z<5000 \mathrm{~km} \mathrm{~s}^{-1}$, within which past surveys are believed to be reasonably complete. The incidence rate drops with increasing velocity, probably because of incompleteness and sensitivity limitations (Braatz et al. 2004; Kondratko et al. 2006a). Today, there are $\sim 90$ known masers in $A G N$ (published and unpublished) with $\mathrm{cz}<20000 \mathrm{~km} \mathrm{~s}^{-1}$ (Figure 1), plus the maser seen toward quasar J0804+3607 at $z \sim 0.66$ (Barvainis \& Antonucci 2005). For the time being, the internal structure and physical nature of this maser is not known.

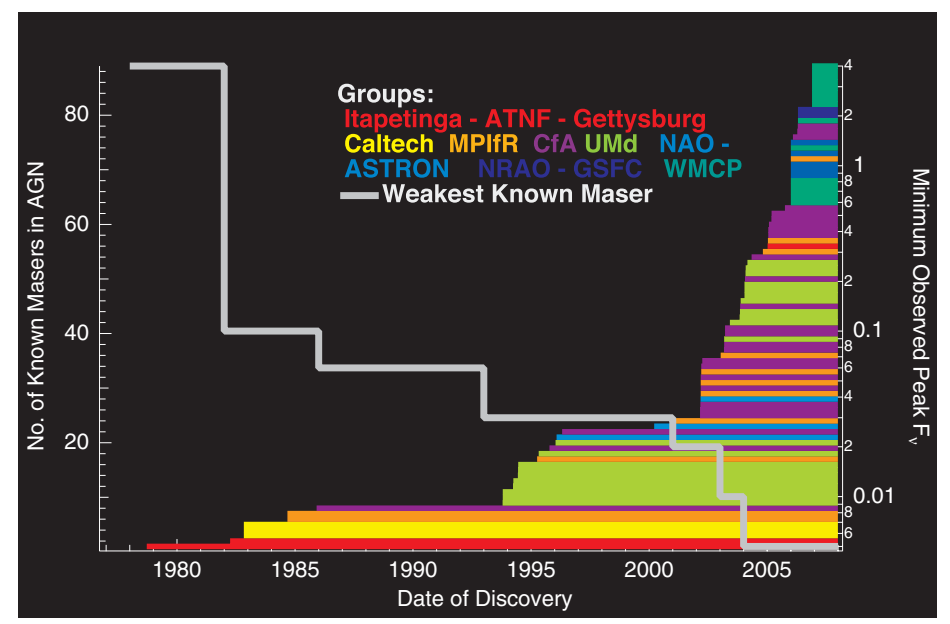

Figure 1. Growth in the number of $\mathrm{H}_{2} \mathrm{O}$ masers believed to lie in AGN (horizontal bars). Color coding associates discoveries with lead institutions. The evolution in the peak flux density of the weakest known maser (down-sloping curve) marks gradual improvement in observing systems.

Because of low detection rates, maser surveys often test stamina, whether comprising shallow integrations on a large number of objects or deep integrations on a small number. The largest surveys have been made with the Green Bank Telescope, NASA Deep Space Network, and Effelsberg 100-m (e.g., Braatz et al. 2004; Greenhill et al. 2003b; Braatz et al. 1996), with important work on smaller samples also completed at Nobeyama (e.g., Nakai et al. 1995). As of now, > 2000 galaxies outside the Local Group had been targeted since the early work by Henkel et al. (1984) and Claussen et al. (1984). † Another 660 ostensibly normal galaxies have been observed as well (Braatz, Greenhill, unpublished).

The broadest bandwidth maser source known covers $\sim 2600 \mathrm{~km} \mathrm{~s}^{-1}$ (Figure 2), centered within $\sim 100 \mathrm{~km} \mathrm{~s}^{-1}$ of the host galaxy systemic velocity $\left(V_{\text {sys }}\right)$, and the weakest known peak source flux densities are on the order of $10 \mathrm{mJy}$, which reflects the detection

$\dagger$ See www.cfa.harvard.edu/wmcp/surveys/survey.html for cumulative lists of nondetections. 
thresholds of existing observing systems (Figure 1). Because it is impossible to know $a$ priori the breadth or velocity of the peak maser emission from any particular galaxy, successful observing systems combine high sensitivity and broad bandwidth (e.g., Greenhill et al. 2003b). Preponderance of narrow-band observing systems up to 2001, posed an obstacle. Today, the majority of survey targets have been observed with systems capable of detecting source with peak fluxes on the order of 10 mJy over many thousand $\mathrm{km} \mathrm{s}^{-1}$.

Commonplace qualitative designations are: "kilomaser," "megamaser," and "gigamaser." These are used loosely in the literature (e.g., the so-called gigamaser in TXS 2226-182 is apparently only $10 \times$ more luminous than the common megamaser, e.g., NGC 3079), and classification is based on apparent integrated luminosity relative to that observed in local high-mass star forming regions.

Prefixes will not be used here because of implicit ambiguity. Specifically, maser emission is always beamed, and apparent luminosity $\left(\mathrm{L}_{\mathrm{H}_{2} \mathrm{O}}\right)$ is computed assuming isotropic emission. It may exceed true luminosity by orders of magnitude. The ratio between the two, the beam solid angle, is determined at least in part by source geometry and may differ widely from source to source (e.g., a thin accretion disk beaming into a plane; a thick fragmented disk beaming emission into a squat cylinder; a foreground cloud amplifies jet emission along a pencil beam). Consequently, correlations between various quantities and apparent maser luminosity may be anticipated to exhibit substantial scatter (e.g., $\mathrm{L}_{\mathrm{H}_{2} \mathrm{O}}$ vs. L(2-10 keV), Kondratko et al. 2006b; $\mathrm{L}_{\mathrm{H}_{2} \mathrm{O}}$ vs. X-ray absorption column density, Zhang et al. 2006). Prospectively, the discovery of any tight correlations with $\mathrm{L}_{\mathrm{H}_{2} \mathrm{O}}$ would be notable, suggesting commonalities among underlying physical systems. However, absent this, qualitative (loose) correlations are still valuable, in particular an apparent transition at $\mathrm{L}_{\mathrm{H}_{2} \mathrm{O}}=1-10 \mathrm{~L}_{\odot}$ between excitation by star formation and by nuclear activity (Henkel et al. 2005).

\section{Spectroscopy}

Because VLBI follow-up is time consuming, working physical classification of maser systems is often made based on the morphology of spectra.

- High-velocity - distinct red and blue-shifted emission complexes approximately symmetrically offset from $V_{\text {sys }}\left(\left|\mathrm{V}-\mathrm{V}_{\text {sys }}\right| \gtrsim 200 \mathrm{~km} \mathrm{~s}^{-1}\right)$. Perceived degrees of symmetry vary from source to source depending on the density of Doppler components. Emission close to $V_{\text {sys }}$ may also be visible, offset by $\lesssim 10 \%$ of the total source breadth. Spectra that include high-velocity and systemic emission complexes are distinctive indicators of emission from highly inclined accretion disks (Figure 2).

- Low-velocity/broad - emission broader than $\sim 100 \mathrm{~km} \mathrm{~s}^{-1}$, peaking within $\sim 300 \mathrm{~km} \mathrm{~s}^{-1}$ of $V_{\text {sys }}$ (Figure 3 ). These masers are circumstantially associated with jet activity based on, thus far, a small sample.

- Low-velocity/narrow - emission comprising a small number of narrow Doppler components within $\sim 100 \mathrm{~km} \mathrm{~s}^{-1}$ of $\mathrm{V}_{\text {sys }}$. The types of the underlying physical systems in these cases are uncertain.

- Ambiguous - Doppler components distributed in velocity, including what may be high-velocity emission, but without apparent symmetry (Figure 3). The number of components may be limited, which is one element that makes pattern matching difficult.

All "high-velocity" maser systems that have been studied thus far with very long baseline interferometry (VLBI) trace highly inclined accretion disks or disk-structures whose rotation curves suggest central dynamical masses of $10^{6}$ to $10^{8} \mathrm{M}_{\odot}$. These include NGC 4258 (Miyoshi et al. 1995), NGC 1068 (Greenhill \& Gwinn 1997), Circinus (Greenhill et al. 2003a), NGC 3079 (Trotter et al. 1998; Yamauchi et al. 2004; Kondratko 


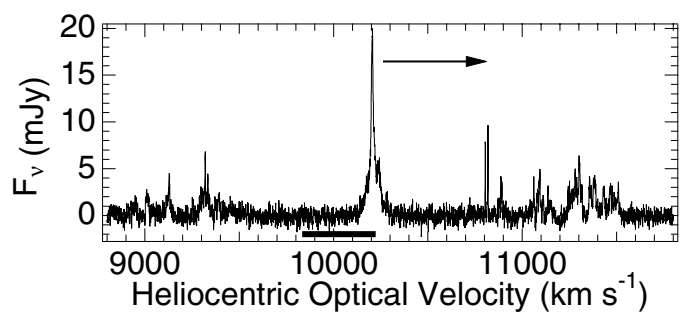

Figure 2. Distinct red and blue-shifted emission complexes, symmetry, and secular drift (arrow) in the UGC 9618B maser. All three are spectroscopic signatures of emission from edge-on disks. A bar along the velocity axis indicates the range of published $\mathrm{V}_{\text {sys }}$ (Kondratko et al. 2006b).
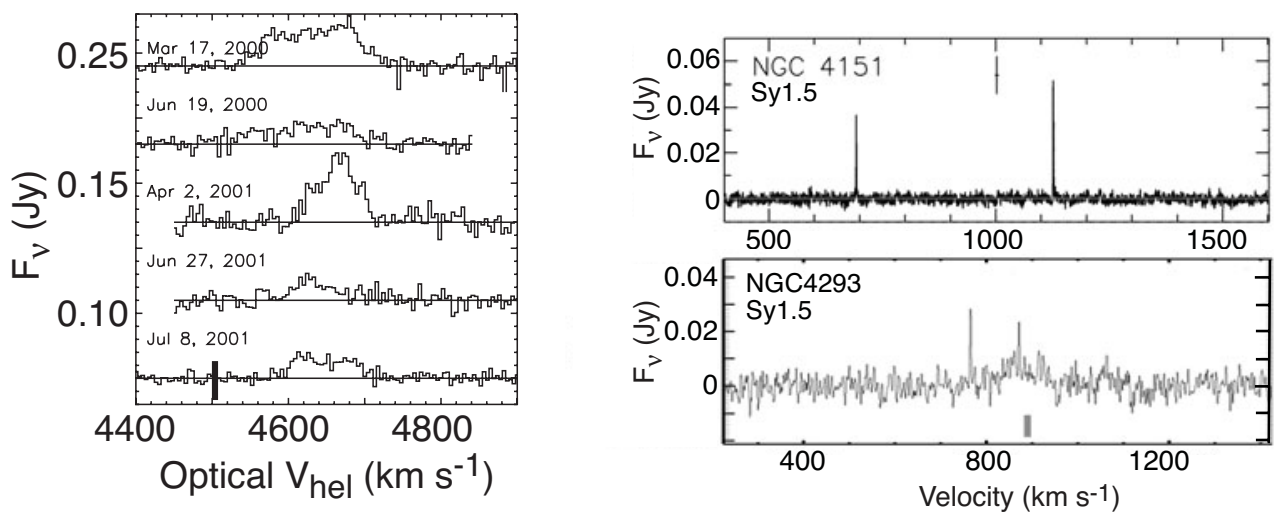

Figure 3. (left) - The Mrk348 maser is associated with jet activity and dominated by broad emission features, an empirical signature of the origin (Peck et al. 2003). (right top)-A maser with ambiguous classification; LSR/optical velocity (Braatz et al. 2004). (right bottom) - a low-velocity/narrow maser; heliocentric/radio velocity(Kondratko et al. 2006a). Bars mark $\mathrm{V}_{\text {sys }}$.

et al. 2004), NGC 3393 (Kondratko et al. 2007), IC 2560 (Ishihara et al. 2001; Greenhill et al. in prep.), and NGC 6323 and UGC 3789 (Braatz, this volume). Three broad lowvelocity systems that have been mapped are offset from the central parsec and are seen toward radio jets (NGC 1068, Gallimore et al. 1996, 2004; NGC 1052, Claussen et al. 1998; Mrk 348, Peck et al. 2003).

Supplemental classifications are also used to clarify uncertain identifications.

- Accelerating - long-term redward secular drift of Doppler components close to $V_{\text {sys }}$. This has been linked to orbital centripetal acceleration within inclined disks.

- Symmetric - emission extending $\gtrsim 200 \mathrm{~km} \mathrm{~s}^{-1}$ (symmetrically) to either side of $\mathrm{V}_{\text {sys }}$, though perhaps without separable emission complexes. This also marks disks.

- Low-luminosity - apparent luminosities $\lesssim 10 \mathrm{~L}_{\odot}$. Low luminosity often appears to be associated with maser action driven by star formation.

For instance, lacking high-velocity emission, the NGC 2639 maser classification would be "Ambiguous" but for observed secular drift that suggests the maser emission originates in a disk (Wilson et al. 1995). The NGC 1068 maser exhibits chiefly plateau emission, but symmetry in maximum velocity offsets of $\pm 330 \mathrm{~km} \mathrm{~s}^{-1}$ is a directly attributable to origin in a disk (Greenhill \& Gwinn 1997). Lastly, the M 82 maser spectrum exhibits just a few Doppler components near $\mathrm{V}_{\text {sys }}$, but low apparent luminosity suggests origin in star formation (Ho et al. 1987), as confirmed by direct imaging (Hagiwara 2007). These are "static" examples. In contrast, spectra may develop defining characteristics due to natural source variability; an "ambiguous" maser with no high-velocity emission at one epoch may 
display symmetric high-velocity emission at a later epoch (e.g., NGC 3735, Kondratko et al., in prep). Such cases reinforce the importance of monitoring in spectroscopic studies.

\section{Mapping}

Water maser emission traces underlying dense, warm gas. The details of amplification in any individual parcel of gas may be difficult to quantify, but nonetheless each observable maser spot samples line-of-sight velocity on the sky, marks the sky position of a stationary point along the line of sight in the line-of-sight velocity field, and points to volumes where energy is deposited into the molecular gas. Necessarily, sampling is incomplete because maser emission is beamed (i.e., any one observer will see only a fraction of masers in a given source). However, inferences from the distribution of maser positions and velocities, coupled with a priori constraints on geometry (e.g., largely planar, point-symmetric, rotating structures, with circular or nearly circular orbits), enable construction of robust models and meaningful 3D deprojections.

For a nearly edge-on, differentially rotating, well ordered, thin $(h / r \lesssim 0.1)$, flat disk that is heated by irradiation broadly across its surface or internally via viscous dissipation, velocity coherence favors $\mathrm{H}_{2} \mathrm{O}$ maser emission: (1) in a narrow sector on the near/far side, and (2) close to the disk-diameter perpendicular to the line of sight (the "midline"). The former gives rise to spectral features near the systemic velocity (Figure 2). The latter gives rise to high-velocity emission that traces the rotation curve of the disk when mapped with interferometers. NGC 4258 is the archetype in this respect (Herrnstein et al. 2005; Moran, this volume).

Emission loci for thick disks $(h / r \gtrsim 0.1)$ are comparatively difficult to predict. NGC 3079 is a good example (Kondratko et al. 2004). Red and blue-shifted emission are segregated on opposite sides of the central engine, but otherwise the maser structure is clumped with local velocity dispersions $>10 \%$ of the rotation speed. Between the two extremes are the relatively well-ordered mid-range cases of Circinus, which is somewhat clumpy but Keplerian to within modelling errors (Greenhill et al. 2003b), and NGC 1068, which is moderately clumpy and distinctly sub-Keplerian (Greenhill \& Gwinn 1997; Lodato \& Bertin 2003).

Warps also complicate prediction of emission loci. Maser photons are beamed close to disk planes, (probably) with narrower beam angles for thinner disks. Warps broaden the solid angle into which emission is beamed but and separate the loci where line-of-sight velocity is coherent and where the disk is seen edge-on. Despite variation of $\sim 0.1 \mathrm{rad}$ in Euler angles over a 2:1 range of radius, the high-velocity emission loci in NGC 4258 are dictated by velocity coherence rather than local tangency of the line of sight to the disk. This could be attributed to finite disk thickness, where in the limiting case of a paper-thin disk, the reverse would be true.

\section{Key science}

Key science directly enabled by study of disk masers follows two principal threads (Figure 4): study of AGN structure (e.g., Morganti et al. 2004) and "maser cosmology," i.e., characterization of cosmological parameters via estimation of accurate geometric distances to maser host galaxies (Greenhill 2004; also Braatz, this volume). Notably, the latter depends on the former in that estimation of distances requires clean 3D dynamical models of maser disks, obtained via fitting to maser positions and line-of-sight velocities.

AGN Structure- Very long baseline study of disk masers is the only means by which structures $<1$ pc from massive black holes can be directly mapped. A 1 pc diameter annulus of maser emission at a distance of $100 \mathrm{Mpc}$ subtends on the order of 10 interferometer 


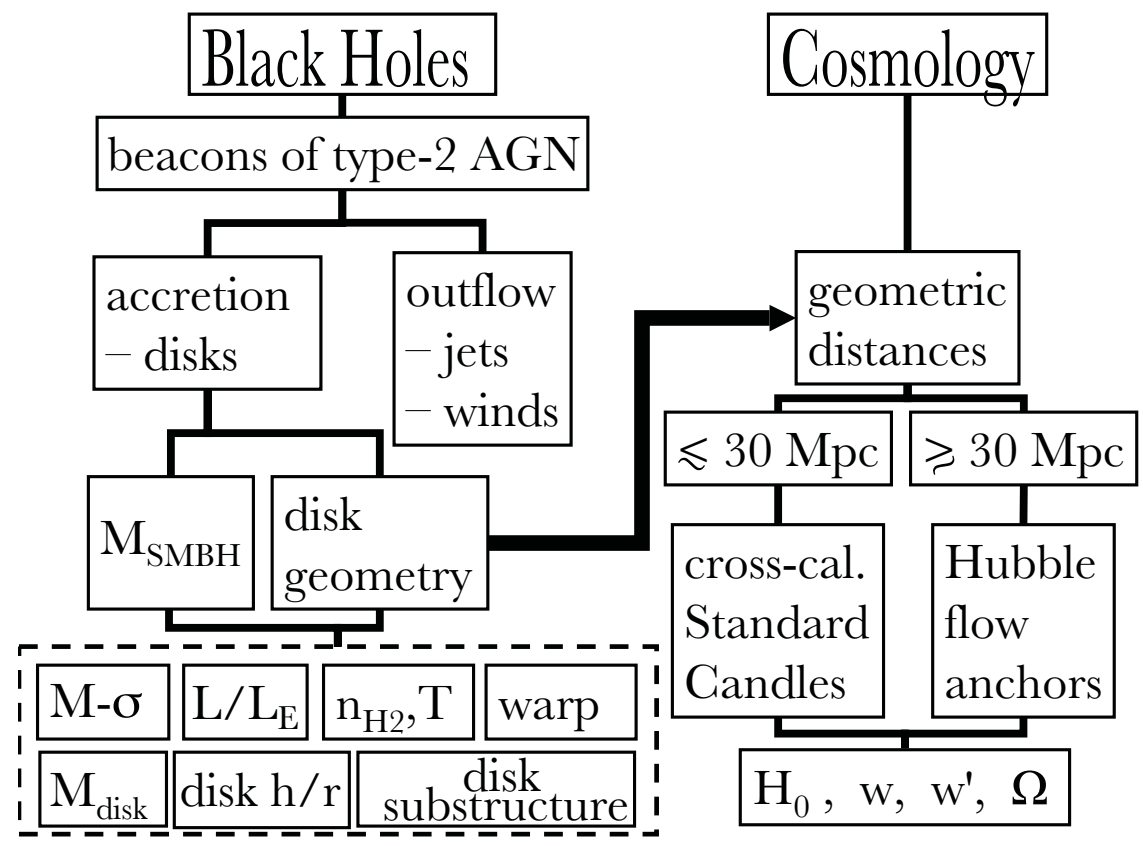

Figure 4. Two threads in the science that may be accomplished through study of $\mathrm{H}_{2} \mathrm{O}$ masers in AGN. Water masers are quite often beacons of activity in galactic nuclei. Related science impacts studies of AGN physics and refinement of the extragalactic distance scale, with ramifications for estimation of cosmological parameters.

beams; a maser that is $2000 \mathrm{~km} \mathrm{~s}^{-1}$ across can be resolved into at least as many spectral channels with VLB correlators. Mapping disks in type-2 AGN can be particularly valuable because obscuration complicates the spectroscopic and timing analyses of central engines at other wavebands, and high inclinations superpose reflected, scattered, and partially obscured emission from the central engines and surrounding media with a range of temperatures. Effectively, the AGN that are the most difficult to study with existing instruments at other wavebands are amenable to detailed radio study when maser emission is present. The contrast is perhaps strongest for AGN with Compton thick obscuration, for which disk masers have a strong preference (Figure 5).
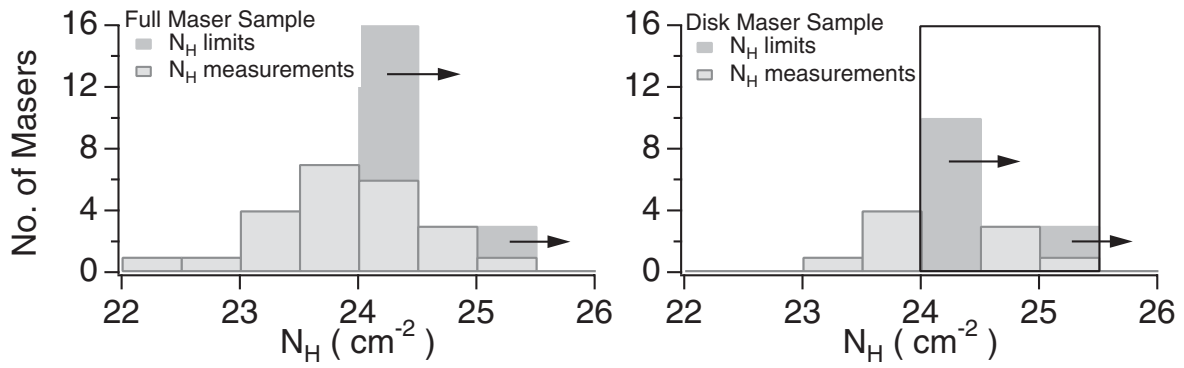

Figure 5. Relatively high incidence of disk borne maser emission among heavily obscured AGN. (left) - Histogram of known X-ray absorption columns for maser AGN. (right) - Subset believed to originate in disks, based on evidence from spectroscopy or mapping. Columns are Compton thick in 16 of 21 cases (heavy-lined box). See Madejski et al. (2006) and Zhang et al. (2006). 
Modelling VLB data in principal enables accurate measurement of central engine dynamical masses, disk masses, Eddington luminosities, and descriptive ratios such as $\mathrm{L}_{\text {Edd }} / \mathrm{L}_{\mathrm{bol}}$. Dynamical masses may be used to refine $\mathrm{M}_{\bullet}-\sigma$ and $\mathrm{M}_{\bullet}-M_{\text {gal }}$ relations (e.g., Ferrarese \& Merritt 2000; Ferrarese et al. 2006) that address the question: what are the roles of massive black holes in galaxy formation? As well, detailed maps of disk structure may be used to assess the balance among central engine gravity, disk selfgravity, luminosity, and dissipation (e.g., Tilak et al. 2008). Independent estimation of disk systemic velocities is also possible, enabling refinement of galactic systemic velocities, or estimation of central engine drift (cf. Reid \& Brunthaler 2004). In the case of warped disks, orientations are known to change by $0.1-1$ rad over radii of $0.1-1$ pc. Misalignment between central engine principle axes and reservoirs of accreting material on parsec and larger scales can be significant (e.g., NGC 3079; Kondratko et al. 2004). Quantitative characterization of warps enables assessment of torque origins and disk stability (e.g., Maloney et al. 1996). This also enables assessment of warped disks as the origin of obscuring columns in at least some, with ramifications for the unification paradigm. The suggestion is borne out for NGC 4258 (Fruscione et al. 2005; Herrnstein et al. 2005), and circumstantial evidence for Circinus supports the hypothesis that shadowing by warps may in some cases bound outflows and photoionized regions (Greenhill et al. 2003a).

Cosmology - The Standard Model is flat and comprises radiation, baryonic matter, non-baryonic matter, and dark energy. It has been defined largely by analyses of Cosmic Microwave Background fluctuations and supernova distances (e.g., Spergel et al. 2007). Nevertheless, estimates of geometry and the physical nature of dark energy remain too uncertain to distinguish among contending theories without substantive assumptions; external constraints are required, as from S-Z effect or lensing. High-accuracy measurement of the Hubble constant, $H_{\circ}$, would be another and arguably a more direct one. Accuracy of $\sim 1 \%$ would enable strong constraint on the dark energy equation of state $(\mathrm{Hu} 2005$; Macri et al. 2006). The present best independent estimate of $H_{\circ}$ is perhaps accurate to $10 \%$, though systematic errors are difficult to quantify (Argon et al. 2007).

A sample of a few to a few tens of anchor galaxies with measured geometric distances could support direct estimation of $\mathrm{H}_{\circ}$, probably with higher accuracy than is possible with traditional Standard Candles, considering intrinsic and systematic uncertainties. All distance estimates for $N$ anchors being equally good, uncertainties in $H_{\circ}$ would scale as $N^{-0.5}$ because systematic uncertainties for disk models among galaxies depend chiefly on individual emission distributions and are uncorrelated. In fact, focus on a few very good cases, e.g., NGC 4258-like galaxies in the Hubble flow, might yield better results overall than a larger sample of more middling cases, though the distribution in $c z$ matters.

In principal, two geometric distance estimates may be obtained for each maser galaxy, one via analysis of centripetal acceleration, and one via analysis of proper motion (Herrnstein et al. 1999). Both observables are greatest for masers close to the systemic velocity. Measurement of acceleration is easier and less resource intensive. (Proper motions are a very small fraction of a beamwidth for earth-bound baselines; $1000 \mathrm{~km} \mathrm{~s}^{-1}$ subtends $\sim 2 \mu$ as yr $^{-1}$ at $\sim 100 \mathrm{Mpc}$, or $\sim 1 \%$ of a beam at $\lambda 1 \mathrm{~cm}$. For an orbital radius of $1 \mathrm{pc}$, this speed corresponds to a $\sim 1 \mathrm{~km} \mathrm{~s}^{-1} \mathrm{yr}^{-1}$ drift or about one line width per year.) Nonetheless, VLB study is requisite to establish a disk rotation curve and model from which to estimate central mass divided by distance and deprojected orbital radii for systemic emission. The latter are distinct from the radii of high-velocity emission inferred from rotation curves and in first order analyses may be inferred from the rate of change of line-of-sight velocity as a function of position along the disk plane. 
The basic methodology to measure the geometric distance to an anchor galaxy involves several VLB epochs, coupled with single-dish monitoring of spectra over a time interval that includes the VLB observations (to enable cross-referencing). Considering time variability of Doppler components over weeks to months, VLB epochs need to be closely spaced in time (in the event more than one is required to achieve adequate sensitivity). However, widely spaced clusters of VLB epochs are also useful in cases where the velocity pattern of Doppler components evolves (i.e., new lines appearing at new velocities). Repeated VLB observation may broaden the fraction of the disk that can be traced (e.g., by sampling the midline over a larger range of radii), thus improving model quality.

VLB modelling efforts thus far suggest the best and highest priority masers for estimation of extragalactic distances would be those that conform to five basic criteria:

- red and blue-shifted high-velocity emission -

o many high-velocity lines sampling rotation curves over a broad range of radius;

- emission close to the systemic velocity -

o many lines near systemic that display measurable centripetal accelerations;

- measurable position-velocity gradients from which radii may be inferred;

- thin disk structure -

o emission divisible by eye into narrow Doppler components;

- Keplerian rotation (i.e., little evidence for fragmentation or other perturbation);

o a rich emission distribution that enables robust modelling, including independent estimation of disk inclination, systemic velocity, and dynamical center location;

- peculiar motion that is small with respect to total recessional velocity $\circ$ origin in the Hubble Flow;

- a line strong enough for VLB self-calibration or a strong calibrator $\ll 1^{\circ}$ away.

\section{References}

Argon, A. L., Greenhill, L., Reid, M., Moran, J., \& Humphreys, E. 2007, ApJ 659, 1040

Baan, W. A. \& Klöckner, H.-R. 2006, A\& $A$ 449, 559

Barvainis, R. \& Antonucci, R. R. J. 2005, ApJ 628, 89

Braatz, J. A., Wilson, A. S., \& Henkel, C. 1996, ApJS 106, 51

Braatz, J. A., Henkel, C., Greenhill, L. J., Moran, J. M., \& Wilson, A. S. 2004, ApJ 617, L29

Churchwell, E., Witzel, A., Pauliny-Toth, I., Sieber, W., Huchtmeier, W., Roland, J. 1977, A\&A 54,969

Claussen, M. J., Heiligman, G. M., \& Lo,K.-Y. 1984, Nature 310, 298

Claussen, M. J. \& Lo, K.-Y. 1986, ApJ 308, 592

Claussen, M. J., Reid, M. J., Schneps, M. H., Lo, K.-Y., Moran, J. M., \& Güsten, R. 1988, in: M. J. Reid \& J. M. Moran (eds.), The Impact of VLBI on Astrophysics and Geophysics, Proceedings of the 129th IAU Symposium (Dordrecht: Kluwer) vol. 129, p. 231

Claussen, M. J., Diamond, P. J., Braatz, J. A., Wilson, A. S., Henkel, C. 1998, ApJ 500, L129

Dos Santos, P. M. \& Lépine, J. R. D. 1979, Nature 278, 34

Ferrarese, L., et al. 2006, ApJ 644, L21

Ferrarese, L. \& Merritt, D. 2000, ApJ 539, L9

Fruscione, A., Greenhill, L. J., Filippenko, A. V., Moran, J. M., Herrnstein, J. R., Galle, E. 2005, ApJ 624, 103

Gallimore, J. F., Baum, S. A., O’Dea, C. P., Brinks, E., Pedlar, A. 1996, ApJ 462, 740

Gallimore, J. F., Baum, S. A. \& O'Dea, C. P. 2004, ApJ 613, 794

García-Burillo, S., Combes, F., Usero, A., Graciá-Carpio, J. 2007, New Astron. 51, 160

Gardner, F. F. \& Whiteoak, J. B 1982, MNRAS 201, P13

Greenhill, L. J., Jiang, D., Moran, J., Reid, M., Lo, K.-Y., Claussen, M. 1995, ApJ 440, 619

Greenhill, L. J. \& Gwinn, C. R. 1997, ApESSS 248, 261

Greenhill, L. J. 2002a, in: V. Migenes and M. J. Reid (eds.), Cosmic Masers: from Proto-Stars to Black Holes, Proceedings of IAU Symposium 206, (San Francisco: ASP) vol. 206, p. 381 
Greenhill, L. J., et al. 2002b, ApJ 565, 836

Greenhill, L. J., et al. 2003a, ApJ 590, 162

Greenhill, L. J., Kondratko, P. T., Lovell, J. E. J., Kuiper T. B. H., Moran, J. M., Jauncey, D. L., Baines, G. A. 2003b, ApJ 582, L11

Greenhill, L. J. 2004, New Astron. 48, 1079

Hagiwara, Y. 2007 AJ 133, 1176

Henkel, C., Güsten, R., Downes, D., Thum, C., Wilson, T., Biermann, P. 1984, A 6 A 141, L1

Henkel, C., Whiteoak, J. B., Nyman, L.-A., Harju, J. 1990, A 8 A 230, L5

Henkel, C., Peck, A. B., Tarchi, A., Nagar, N. M., Braatz, J. A., Castangia, P., Moscadelli, L. 2005, A\&A 436, 75

Herrnstein, J. R., et al. 1999, Nature 400, 539

Herrnstein, J. R., Moran, J. M., Greenhill, L. J., \& Trotter, A. S. 2005, ApJ 629, 719

Ho, P. T. P., Martin, R. N. Henkel, C., Turner, J. L. 1987, ApJ 320, 663

Hu, W. 2005, in: S. Wolff and T. Lauer (eds.) Observing Dark Energy (San Francisco: ASP) vol. 339 , p. 215

Humphreys, E. M. L., et al. 2005, ApJ 634, L133

Ishihara, Y., Nakai, N., Iyomoto, N., Makishima, K., Diamond, P. Hall, P. 2001, PASJ 53, 215

Klöckner, H.-R. \& Baan, W. A. 2005, ApESSS 295, 277

Kondratko, P. T., Greenhill, L. J. \& Moran, J. M., 2004, ApJ 618, 618

Kondratko, P. T., et al. 2006a, ApJ 638, 100

Kondratko, P. T., Greenhill, L. J., \& Moran, J. M. 2006b, ApJ 652, 136

Kondratko, P. T., Greenhill, L. J., \& Moran, J. M. 2007, ApJ submitted

Lépine, J. R. D. \& Dos Santos, P. M. 1977, Nature 270, 501

Lodato, G. \& Bertin, G. 2003, A\&A 398, 517

Macri, L., Stanek, K., Bersier, D., Greenhill, L., \& Reid, M., 2006, ApJ 652, 1133

Madejski, G., Done, C., Życki, P. T., \& Greenhill, L. 2006, ApJ, 636, 75

Maloney, P. R., Begelman, M. C. \& Pringle, J. E. 1996, ApJ 472, 582

Miyoshi, M., Moran, J., Herrnstein, J., Greenhill, L., Nakai, N., Diamond, P., \& Inoue, M. 1995, Nature 373,127

Momjian, E., Romney, J. D., Carilli, C. L., Troland, T. H. 2006, ApJ 653, 1172

Morganti, R., Greenhill, L. J., Peck, A. B., Jones, D. L., Henkel, C. 2004, New Astron. 48, 1195

Nakai, N., Inoue, M. \& Miyoshi, M. 1993, Nature 361, 45

Nakai, N., Inoue, M., Miyazawa, K., Miyoshi, M., Hall, P. 1995, PASJ 47, 771

Peck, A. B., Henkel, C., Ulvestad, J. S., Brunthaler, A., Falcke, H., Elitzur, M., Menten, K. M., Gallimore, J. F. 2003, ApJ 590, 149

Pihlström Y. M., Conway J., Booth R., Diamond P., Polatidis A. 2001, A\&GA 377, 413

Pihlström, Y. M., Baan, W. A., Darling, J., Klöckner, H.-R. 2005a, ApJ 618, 705

Pihlström, Y. M. 2005b, in: J. Romney \& M. Reid (eds.), Future Directions in High Resolution Astronomy, (San Francisco: ASP) vol. 340, p. 224

Reid, M. J. \& Brunthaler, A. 2004, ApJ 616, 872

Richards, A. M. S., et al. 2005, MNRAS 364, 353

Rovilos, E., Diamond, P., Lonsdale, C., Lonsdale, C., Smith, H. 2003, MNRAS 342, 373

Spergel, D. N., et al. 2007, ApJS 170, 377

Tarchi, A., Castangia, P., Henkel, C., Menten, K. M. 2007, New Astron. Revs 51, 67

Tilak, A., Greenhill, L. J., Done, C., Madejski, G. 2008, ApJ submitted

Trotter, A. S., Greenhill, L., Moran, J., Reid, M., Irwin, J., Lo, K.-Y. 1998, ApJ 495, 740

Trotter, A. S., Moran, J., Greenhill, L., Zheng, X., Gwinn, C. 1997, ApJ (Letters) 485, L79

Vignali, C., Brandt, W. N., Comastri, A., Darling, J. 2005, MNRAS 364, 99

Wilson, A. S., Braatz, J. A., \& Henkel, C. 1995, ApJ 455, L127

Yamauchi, A., Nakai, N., Sato, N., Diamond, P. 2004, PASJ 56, 605

Yates J. A., Richards A. M. S., Wright M. M., Collett J. L., Gray M. D., Field D., Cohen R. J. 2000, MNRAS 317, 28

Zhang, J. S., Henkel, C., Kadler, M., Greenhill, L. J., Nagar, N., Wilson, A. S., Braatz, J. A. 2006, A\&A 450, 933 